\title{
Analysis of Flap Mechanism Reliability
}

\author{
Jie Zhou ${ }^{\mathrm{a}}$, Zhili Sun ${ }^{\mathrm{b}}$ and Qinglin Song ${ }^{\mathrm{c}}$ \\ School of Mechanical Engineering and Automation, Northeastern University, Shenyang 110819, \\ China
}

a2459855007@qq.com, ${ }^{\text {bzhlsun@mail.neu.edu.cn, }{ }^{c 2} 284535503 @ q q . c o m}$

\begin{abstract}
Keywords: Flap Mechanism, Simulink Simulation, Comprehensive Error, Reliability Analysis.
\end{abstract}
\begin{abstract}
In this paper, a dynamic simulation model of flap mechanism is established by using Simulink and simultaneous constraint method. Based on this model, the regularity and volatility of kinematic parameters' error is analyzed under the influence of comprehensive error, the position of flap deflection angle maximum deviation and the maximum drive torque are found. Then, under the dynamic analysis, using vector projection method to integrate the amount of wear, and the reliability of flap deflection accuracy and the reliability of flap seizure are calculated by Monte Carlo method under abrasive wear and synthetic error condition.
\end{abstract}

\section{Introduction}

The high reliability integrated design technology of the flap mechanism is one of the key technologies of modern aircraft design. For the flap mechanism, it is not possible to avoid all kinds of damage from material, design, process, and maintenance, and some of the factors such as load parameters, dimensional error and abrasive wear owning apparent random fluctuation character. As a result, many scholars have extensively studied the reliability of the flap mechanism. In terms of flap fault simulation, Huan Pang, Bo Yuan, Lijie Cui et al used the virtual prototyping technology to establish the rigid and flexible coupling model of the flap mechanism, and studied the reliability of flap seizure [1-3].In design of the flap mechanism, Zhisheng Yi ,Bo Yuan, Xiaojun Zhu et al made a qualitative analysis about the relationship among different parameters [4-5]. At the present stage, the researchers just carried out kinematic analysis and virtual simulation of the simple flap mechanism, for the flap mechanism with compound movement, no kinetic model was established and no corresponding reliability analysis was carried out.

\section{Dynamic Modeling based on Joint Constraint Method}

The flap mechanism diagram and simplify model as shown in Fig.1.Here the actuator rotation center $O$ is the coordinate origin, vertical wing chord plane upward is the $Y$-axis positive direction, vertical wing beam outward is the $X$-axis positive direction, $K$ is the centroid of flap, $C$ is the centroid of the carriage, $F$ is the aerodynamic load acting on the centroid of the flap. $w_{1}, w_{2}$ is the horizontal distance and vertical distance between actuator rotation center and the hinge center of last link DE, $w_{3}$ is the distance between the centroid of the carriage and the actuator rotation center. $\theta_{c}$ is the angle between link $B C$ and link $C D, \theta_{k}$ is the angle between link $C K$ and link $C D . \alpha$ is the angle between the slide surface and the wing chord plane. We assume that the positive direction of the X-axis is the starting direction of the component rotation angle $\theta$ and the counterclockwise direction is positive. 

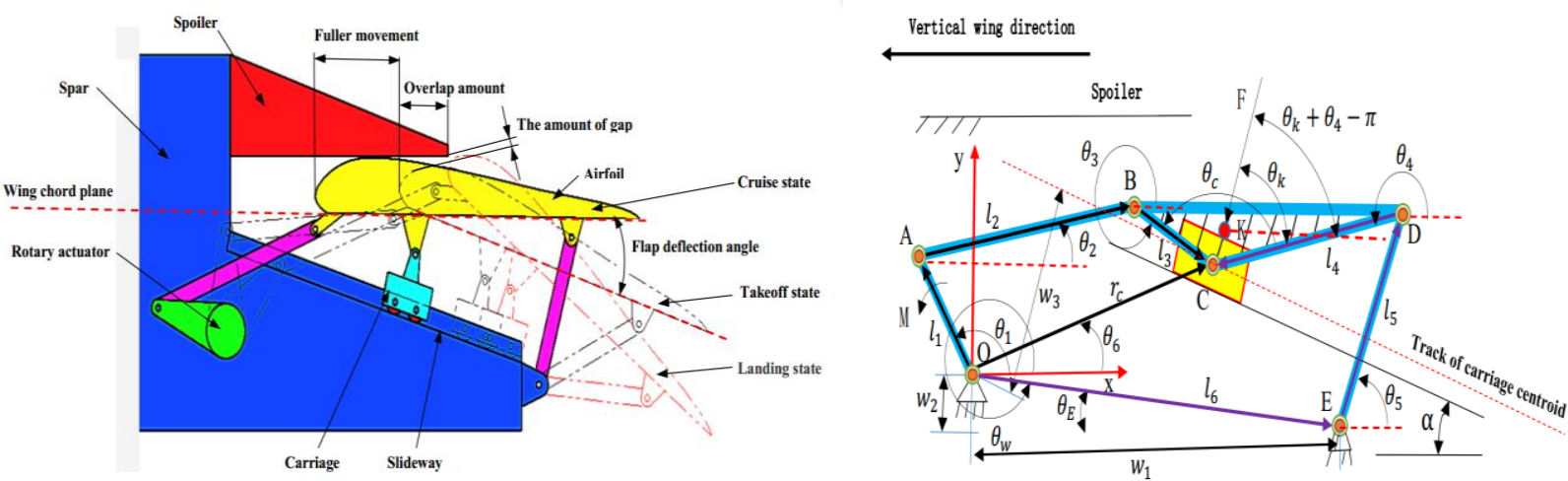

Fig.1 flap mechanism diagram and simplify model

According to the mechanics theory, the system equations of the flap mechanism as follows:

$$
\begin{aligned}
& \begin{array}{c}
F_{61, x}+F_{12, x}-m_{1} a_{c 1, x}=0 \\
F_{61, y}-F_{12, y}-m_{1} a_{c 1, y}=m_{1} g \\
-F_{12, y} l_{1} \cos \theta_{1}-F_{12, x} l_{1} \sin \theta_{1}+M=m_{1} g r_{c 1} \cos \theta_{1}+I_{1} \alpha_{1} \\
F_{12, x}+F_{23, x}-m_{2} a_{c 2, x}=0
\end{array} \\
& -F_{12, y}-F_{23, y}-m_{2} a_{c 2, y}=m_{2} g \\
& F_{12, y} r_{c 2} \cos \theta_{2}+F_{12, x} r_{c 2} \sin \theta_{2}-F_{23, y}\left(l_{2}-r_{c 2}\right) \cos \theta_{2} \\
& -F_{23, x}\left(l_{2}-r_{c 2}\right) \sin \theta_{2}-I_{2} \alpha_{2}=0 \\
& F_{23, x}+F_{34, x}+F_{35, y}-m_{3} a_{c 3, x}=F \cos \left(\theta_{k}+\theta_{4}-\pi\right) \\
& -F_{23, y}-F_{34, y}-F_{35, y}-m_{3} a_{c 3, y}=F \sin \left(\theta_{k}+\theta_{4}-\pi\right)+m_{3} g \\
& -F_{23, x} l_{3} \cos \left(\theta_{3}-1.5 \pi\right)+F_{23, y} l_{3} \sin \left(\theta_{3}-1.5 \pi\right)+ \\
& F_{35, x} l_{4} \sin \left(\pi-\theta_{4}\right) \\
& -F_{35, y} l_{4} \cos \left(\pi-\theta_{4}\right)-I_{3} \alpha_{3}=m_{3} g r_{c 2} \cos \left(\theta_{k}+\theta_{4}-\pi\right) \\
& F_{34, x}+(\sin \alpha-\sigma \mu \cos \alpha) F_{N}-m_{4} \ddot{x}_{\mathrm{c}}=0 \\
& -F_{34, y}+(\cos \alpha+\sigma \mu \sin \alpha) F_{N}-m_{4} \ddot{y}_{c}=m_{4} g \\
& F_{65, x}+F_{35, x}-m_{5} a_{c 5, x}=0 \\
& -F_{35, y}+F_{65, y}-m_{5} a_{c 5, y}=m_{5} g \\
& -F_{35, x} l_{5} \sin \theta_{5}-F_{35, y} l_{5} \cos \theta_{5}-I_{5} \alpha_{5}=m_{5} g r_{c 5} \cos \theta_{5} \\
& -l_{2} \alpha_{2} \sin \theta_{2}-l_{3} \alpha_{3} \sin \theta_{3}-\ddot{x}_{c} \\
& =l_{1} \alpha_{1} \sin \theta_{1}+l_{1} \omega_{1}^{2} \cos \theta_{1}+l_{2} \omega_{2}^{2} \cos \theta_{2}+l_{3} \omega_{3}^{2} \cos \theta_{3} \\
& l_{2} \alpha_{2} \cos \theta_{2}+l_{3} \alpha_{3} \cos \theta_{3}-\ddot{y}_{c} \\
& =l_{1} \alpha_{1} \cos \theta_{1}+l_{1} \omega_{1}^{2} \sin \theta_{1}+l_{2} \omega_{2}^{2} \sin \theta_{2}+l_{3} \omega_{3}^{2} \sin \theta_{3} \\
& -l_{4} \alpha_{4} \sin \theta_{4}-l_{5} \alpha_{5} \sin \theta_{5}-\ddot{x}_{c}=l_{5} \omega_{5}^{2} \cos \theta_{5}+l_{4} \omega_{4}^{2} \cos \theta_{4} \\
& \alpha_{3}-\alpha_{4}=0 \\
& -\tan \alpha \ddot{x}_{c}-\ddot{y}_{c}=0 \\
& a_{c 1, x}=-r_{c 1} \alpha_{1} \sin \theta_{1}-r_{c 1} \omega_{1}^{2} \cos \theta_{1} \\
& a_{c 1, y}=r_{c 1} \alpha_{1} \cos \theta_{1}-r_{c 1} \omega_{1}^{2} \sin \theta_{1} \\
& a_{c 2, x}+r_{c 2} \alpha_{2} \sin \theta_{2}=-l_{1} \alpha_{1} \sin \theta_{1}-r_{c 2} \omega_{2}^{2} \cos \theta_{2}-l_{1} \omega_{1}^{2} \cos \theta_{1} \\
& a_{c 2, y}-r_{c 2} \alpha_{2} \cos \theta_{2}=l_{1} \alpha_{1} \cos \theta_{1}-l_{1} \omega_{1}^{2} \sin \theta_{1}-r_{c 2} \omega_{2}^{2} \sin \theta_{2} \\
& a_{c 3, x}-\ddot{x}_{c}+r_{c 3} \alpha_{3} \sin \left(\theta_{k}+\theta_{4}-\pi\right)=-r_{c 3} \omega_{3}^{2} \sin \left(\theta_{k}+\theta_{4}-\pi\right) \\
& a_{c 3, y}-\ddot{y}_{c}-r_{c 3} \alpha_{3} \cos \left(\theta_{k}+\theta_{4}-\pi\right)=-r_{c 3} \omega_{3}^{2} \sin \left(\theta_{k}+\theta_{4}-\pi\right) \\
& a_{c 5, x}+r_{c 5} \alpha_{5} \sin \theta_{5}=-r_{c 5} \omega_{5}^{2} \cos \theta_{5} \\
& a_{c 5, y}-r_{c 5} \alpha_{5} \cos \theta_{5}=-r_{c 5} \omega_{5}^{2} \sin \theta_{5}
\end{aligned}
$$

Here $m_{i}, l_{i}, I_{i}, \theta_{i}, \omega_{i}, \alpha_{i}$ is the mass, length, moment of inertia, angle, angular velocity and angular acceleration of link $i$ respectively. $r_{c i}$ is the distance between centroid and the center of rotation in link $i . a_{c i, x}, a_{c i, y}$ is the component of the link $i$ centroid acceleration in the $X, Y$ axis. $\ddot{x_{c}} \quad \ddot{y_{c}}$ is the component of the carriage centroid acceleration on the $X, Y$ axis. $M$ is the drive torque. $\mu$ is the rolling friction coefficient between roller and track, $\sigma$ is the carriage movement direction factor and satisfies the following formula:

$$
\sigma= \begin{cases}+1, & \ddot{x}_{c} \geq 0 \\ -1, & \ddot{x_{c}}<0\end{cases}
$$

The above system equations are assembled into matrix form, and the flap mechanism simulation model is established in Simulink as shown in Fig.2. Finally, a program about reliability calculation based on Monte Carlo sampling is written in MATLAB and basic block diagram of the program is shown in Fig.3. 


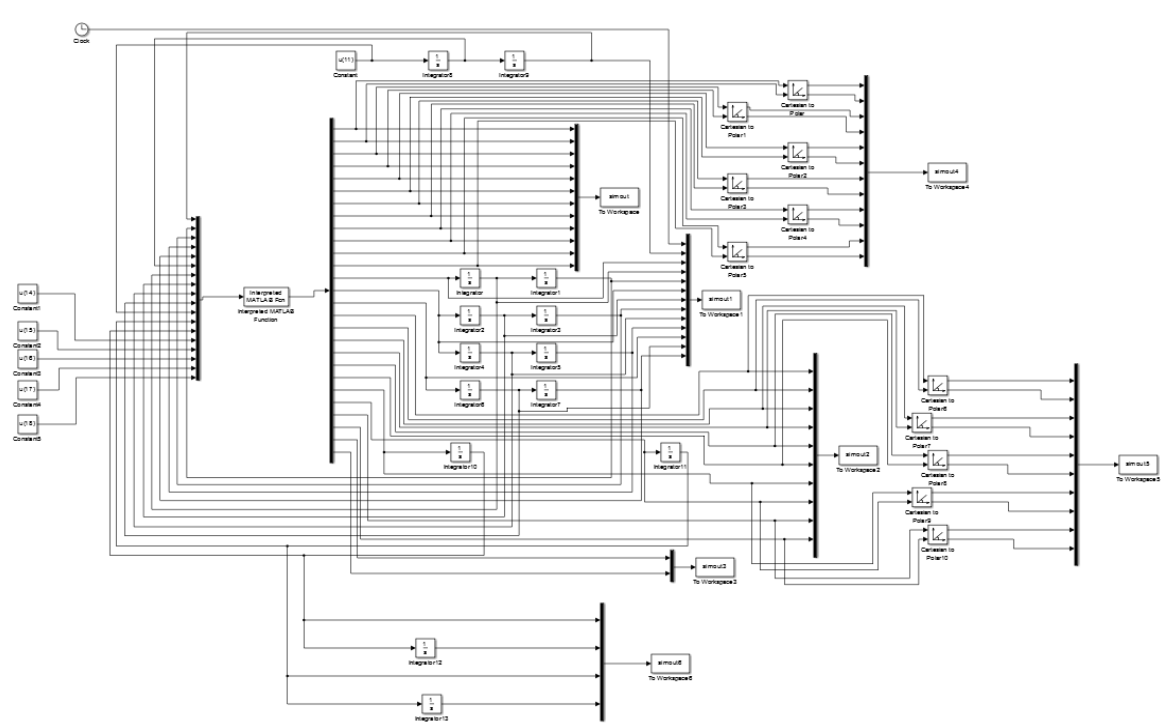

Fig.2 Simulation model of flap mechanism based on Simulink

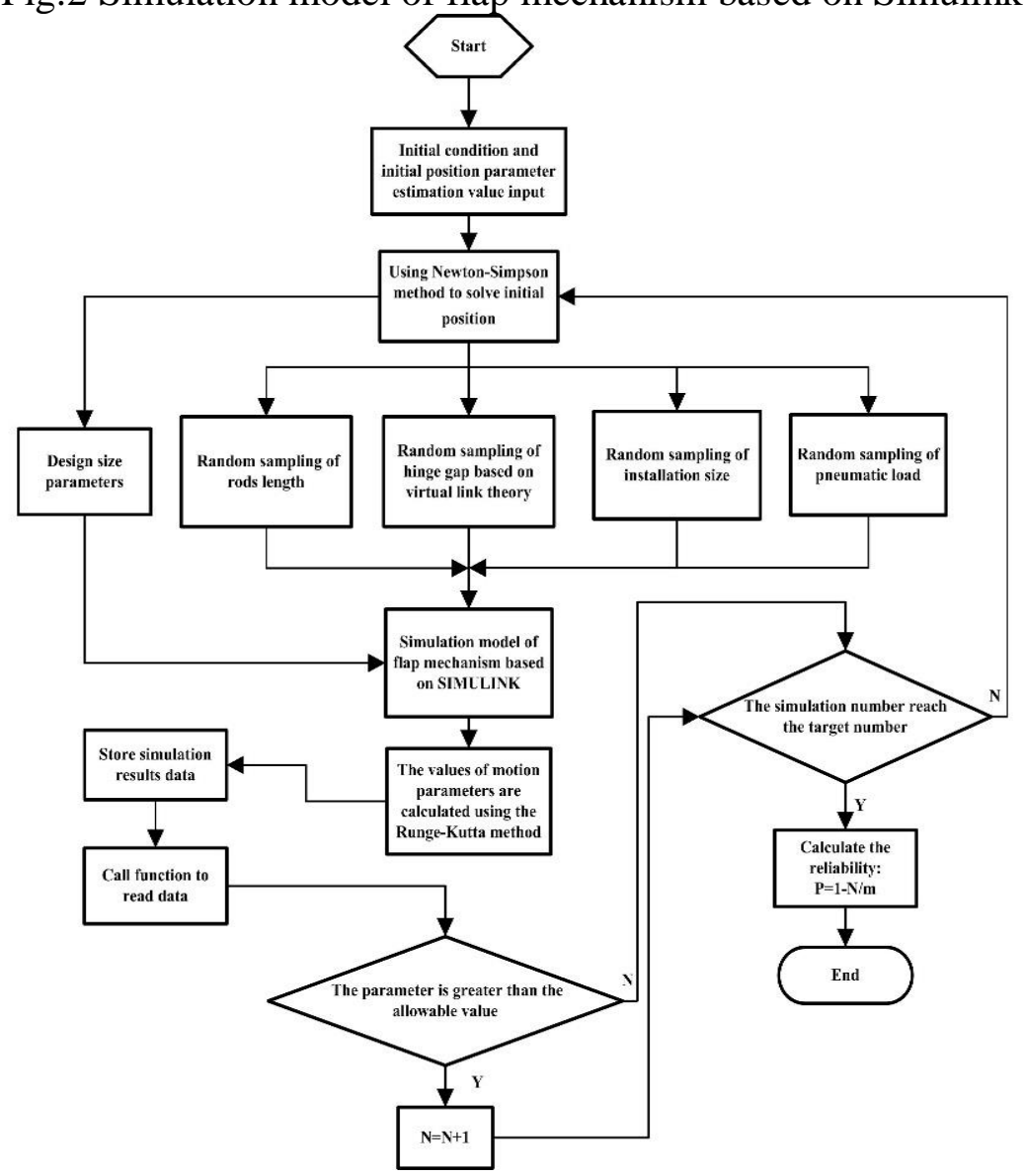

Fig. 3 The block diagram of reliability simulation program

\section{Dynamic Simulation Examples}

\subsection{Initial Parameter Value Assignment}

The values of actual kinematic parameters and design kinematic parameters of the flap mechanism will have a significant deviation due to synthetic error, which include the installation error, size error, hinge clearance, abrasive wear and pneumatic load disturbance. We assume that installation error, size error and aerodynamic loads are subject to normal distribution, and the specific parameters of the flap mechanism are shown in table 1. 
Table 1 parameters list of flap mechanism

\begin{tabular}{|c|c|c|c|c|}
\hline Parameter meaning & Unit & Parameter & $\begin{array}{l}\text { Parameter } \\
\text { value }\end{array}$ & $\begin{array}{l}\text { Parameter } \\
\text { distribution }\end{array}$ \\
\hline \multirow{5}{*}{ The mass of each component } & \multirow{5}{*}{$\mathrm{kg}$} & $m_{1}$ & 2 & \multirow{5}{*}{$\mathrm{NO}$} \\
\hline & & $m_{2}$ & 4 & \\
\hline & & $m_{3}$ & 10 & \\
\hline & & $m_{4}$ & 10 & \\
\hline & & $m_{5}$ & 4 & \\
\hline \multirow{5}{*}{ The length of each link } & \multirow{10}{*}{$\mathrm{m}$} & $l_{1}$ & $0.447 \pm 0.004$ & $l_{1} \sim N\left(0.447,(0.004 / 3)^{2}\right)$ \\
\hline & & $l_{2}$ & $1.070 \pm 0.010$ & $l_{2} \sim N\left(1.070,(0.010 / 3)^{2}\right)$ \\
\hline & & $l_{3}$ & $0.653 \pm 0.006$ & $l_{3} \sim N\left(0.653,(0.006 / 3)^{2}\right)$ \\
\hline & & $l_{4}$ & $1.007 \pm 0.010$ & $l_{4} \sim N\left(1.007,(0.010 / 3)^{2}\right)$ \\
\hline & & $l_{5}$ & $0.700 \pm 0.007$ & $l_{5} \sim N\left(0.700,(0.007 / 3)^{2}\right)$ \\
\hline The pin radius attached to the ith link & & $R_{i}$ & $0.020 \pm 0.001$ & $R_{\mathrm{cxi}} \sim N\left(0.00,(0.001 / 3)^{2}\right)$ \\
\hline The hole radius attached to the jth link & & $r_{j}$ & $0.019 \pm 0.001$ & $R_{\mathrm{cyi}} \sim N\left(0.00,(0.001 / 3)^{2}\right)$ \\
\hline \multirow{3}{*}{ Assembly position parameters } & & $w_{1}$ & $2.010 \pm 0.010$ & $\mathrm{w}_{1} \sim N\left(2.010,(0.010 / 3)^{2}\right)$ \\
\hline & & $w_{2}$ & $0.243 \pm 0.002$ & $w_{2} \sim N\left(0.243,(0.002 / 3)^{2}\right)$ \\
\hline & & $w_{3}$ & $0.648 \pm 0.002$ & $w_{3} \sim N\left(0.648,(0.002 / 3)^{2}\right)$ \\
\hline Angle between link BC and link CD & \multirow{3}{*}{ Deg } & $\theta_{c}$ & 165.32 & NO \\
\hline $\begin{array}{c}\text { Angle between slideway and wing } \\
\text { chord plane }\end{array}$ & & $\alpha$ & $15 \pm 0.1$ & $\alpha \sim N\left(15.000,(0.100 / 3)^{2}\right)$ \\
\hline Angle between line $C K$ and link CD & & $\theta_{k}$ & 105 & NO \\
\hline Aerodynamic load & $\mathrm{N}$ & $\mathrm{F}$ & $15 \pm 1$ & $\mathrm{~F} \sim N\left(15.000,(1.000 / 3)^{2}\right)$ \\
\hline
\end{tabular}

\subsection{Error Analysis of Parameters}

Under the influence of comprehensive error, the parameters in any sampling point will appear random fluctuations. As shown in Fig. 4, the coefficients of variation of the flap deflection angle, angular velocity and angular acceleration are calculated respectively under different simulation times at end position of flap mechanism. We can know that the coefficient of variation fluctuates violently when the number of simulations is less than $8 \times 10^{4}$. But when the number of simulations is bigger than $8 \times 10^{4}$, it begins to stabilize and becomes stable nearly constant.

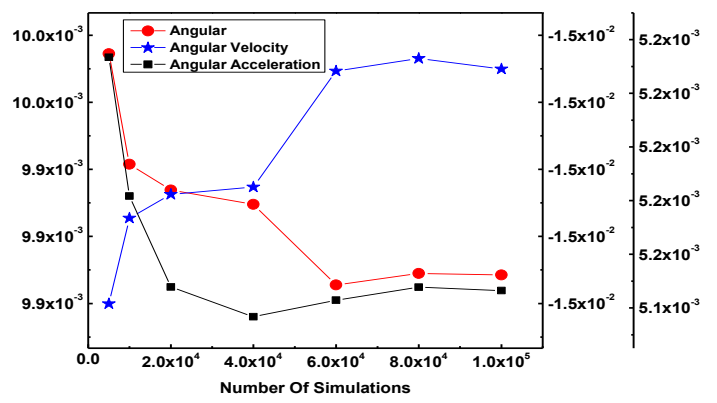

Fig.4 The curve of variation coefficient

As shown in Fig.5, the mean error curve and the standard deviation curve are plotted. The synthetic error has the most significant effect on the flap deflection angle, and the mean error and standard deviation of flap deflection are both two orders of magnitude higher than angular velocity and angular acceleration. The deflection angle mean error and standard deviation reach the maximum at the same time in range of $14^{0}-20^{\circ}$. 

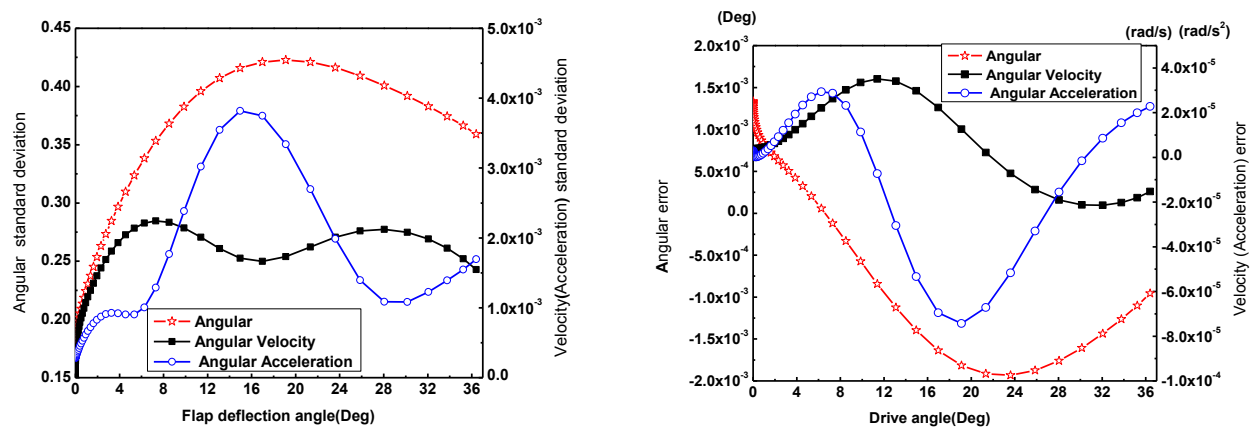

Fig.5The curve of standard deviation and mean error

In order to explore the fluctuation of kinetic parameters, the Monte Carlo sampling method is used to draw the error family curves, as shown in Fig.6. The peak force of the hinge $C$ is three times than the hinge $B$ and $D$, and the angle of contact force is only in range of $-91^{\circ} \sim-97^{\circ}$, so the location of the hinge $\mathrm{C}$ is the position where wear the most intense.
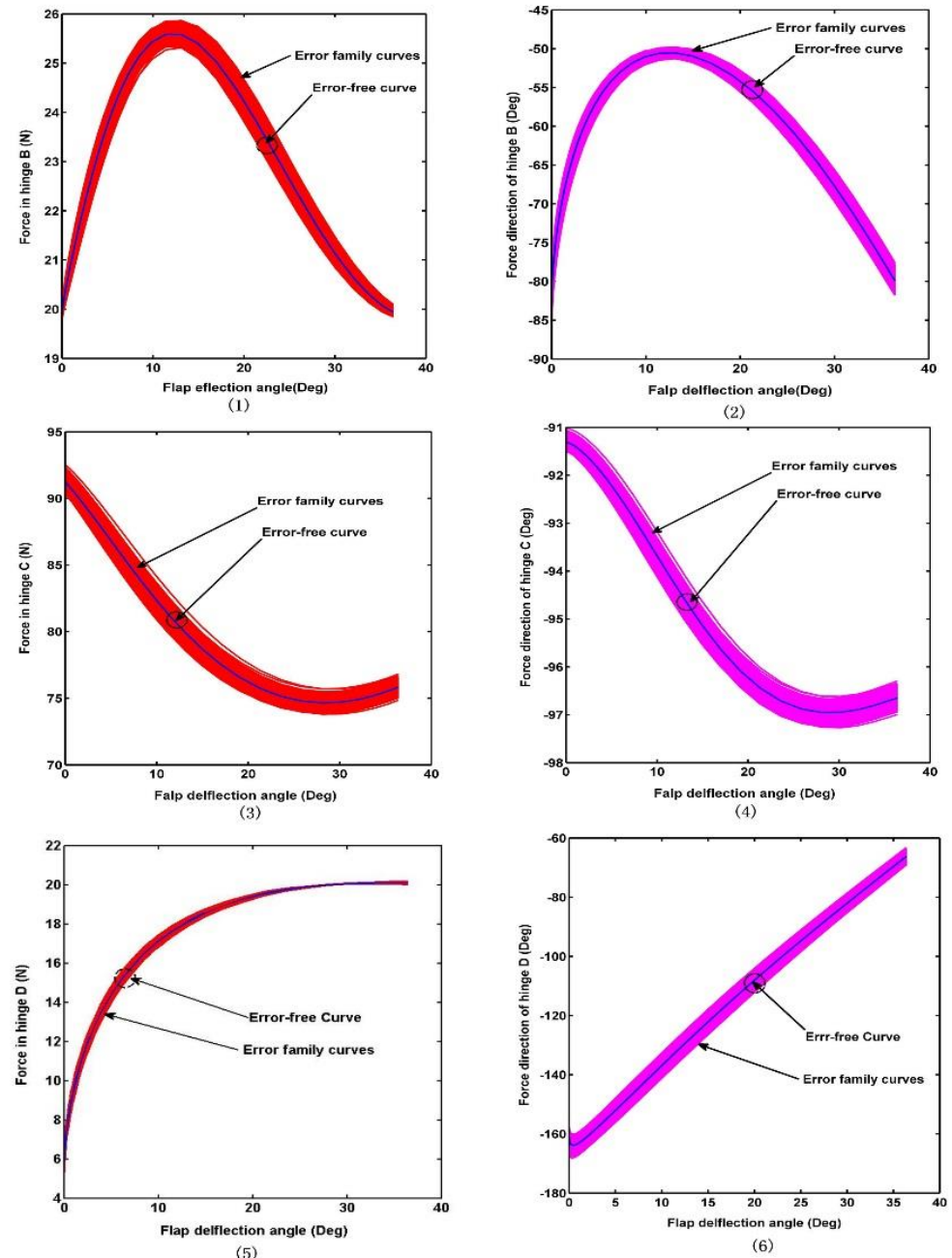

Fig.6 Contact force and direction of Hinge B,C,D

As shown in Fig.7, the peak of the flap track support force is slightly larger than that of the hinge $\mathrm{B}$ and the hinge $\mathrm{D}$, but the error family curve is distributed widely during the whole movement. Take the actual institutions into account, the contact surface prone to fatigue damage under the influence of the fluctuating track support force, so the contact surface of the carriage and the track wear severely. As shown in Fig. 8 the flap mechanism reaches the maximum driving torque when the flap deflection angle in the range of $8^{\circ} \sim 12^{\circ}$. 


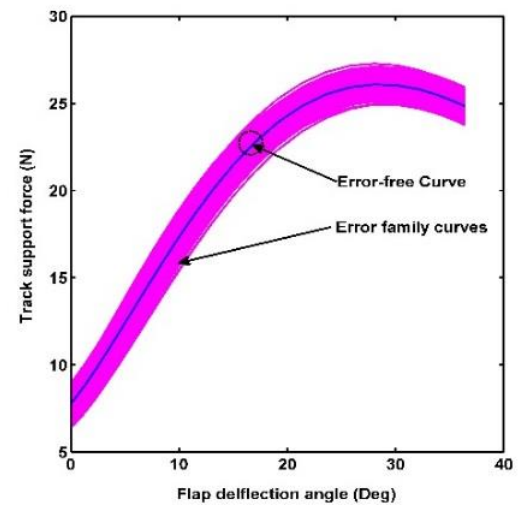

Fig.7 The curve of track support force

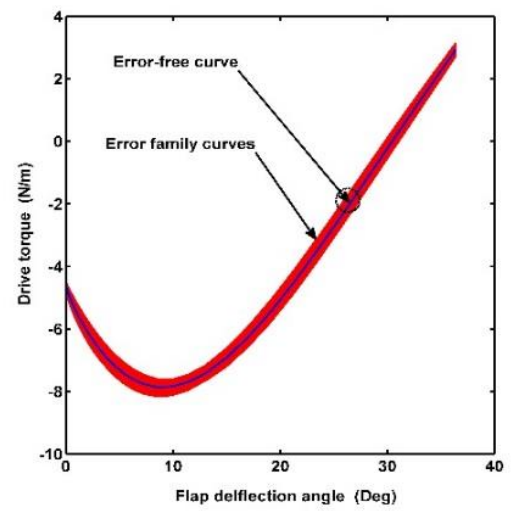

Fig. 8 The curve of drive torque

\section{Reliability Analysis}

According to the dynamic simulation analysis of the flap mechanism, the wear of the flap mechanism mainly exists in hinge $\mathrm{C}$ and the contact surface of the carriage and the track. In this paper, we assume that the wear of roller and track is uniform and equivalent at any position, Due to the angle of contact force is only in the range of $-91^{\circ} \sim-97^{\circ}$, the variation range is extremely small. For convenience sake, the wear vector of hinge $\mathrm{C}$ is projected toward the direction of the wear vector of track and roller, as shown in Fig.9.

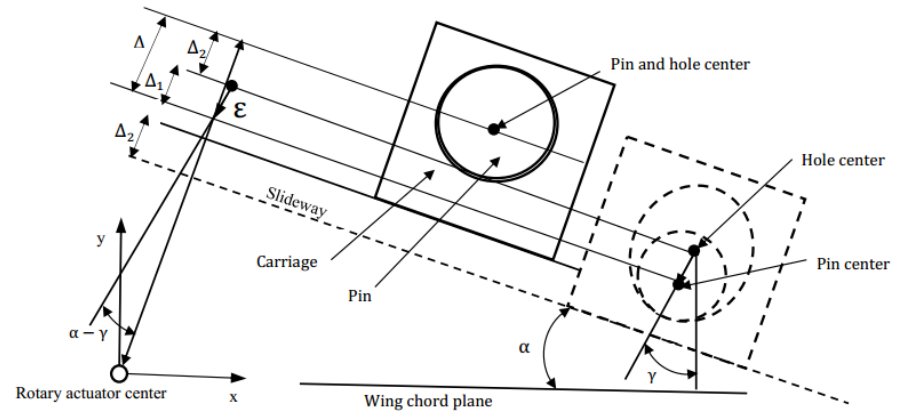

Fig.9 Error vector normalization model

Here, $\alpha$ is the angle between the track plane and the wing chord plane; $\gamma$ is the angle between the wear vector of the hinge $\mathrm{C}$ and the normal direction of the wing chord plane; $e$ is the wear vector of hinge $C ; \Delta_{1}$ is the projection of wear vector of hinge $C$ in the direction of wear vector of track and roller; $\Delta_{2}$ is the track and roller wear; $\Delta$ is the total amount of wear after projection.

\subsection{Reliability Analysis of Flap Deflection Accuracy}

Under dynamic analysis, we can know the flap deflection angle is extremely sensitive to comprehensive error, and the flap angle deviation is maximized in the range of $14^{0}-20^{\circ}$. Therefore, we choose takeoff state to study the reliability of flap deflection accuracy, and the limit state equation as follows:

$$
\mathrm{g}(i)=|| \theta^{0}-\theta_{0}|-| \theta^{(i)}-\theta_{(i)}|| \leq[\Delta \theta](i=0,1,23 \ldots \mathrm{m})
$$

Here, $m$ is the Monte Carlo simulation numbers; $\theta_{(i)}$ is the angle between the initial position of flap and the wing chord plane under the i-th sampling condition; $\theta^{(i)}$ is the angle between the takeoff position of flap and the wing chord plane under the $\mathrm{i}$-th sampling condition; $[\Delta \theta]$ is the allowable flap deflection error.

In order to ensure the flap mechanism cannot occur stuck, we assume the friction coefficient between track and roller is stable under different wear conditions. At $\mathrm{u}=0.05, \mathrm{~m}=100000,[\Delta \theta]=$ $1.5^{\circ}$, the reliability of flap deflection accuracy is calculated and the results are shown in Table 2. 
Table 2. Reliability of flap deflection accuracy $(\mu=0.05)$

\begin{tabular}{ccccc}
\hline Total wear & Parameter & Mean & Standard deviation & Reliability \\
\hline$\Delta=0.000$ & & 0.648 & & 0.99995 \\
$\Delta=0.004$ & & 0.644 & & 0.99915 \\
$\Delta=0.008$ & & 0.640 & $0.002 / 3$ & 0.98090 \\
$\Delta=0.012$ & $\mathrm{w}_{3}$ & 0.636 & & 0.84414 \\
$\Delta=0.016$ & & 0.632 & & 0.48511 \\
$\Delta=0.020$ & & 0.628 & & 0.14292 \\
\hline
\end{tabular}

\subsection{Reliability Analysis of Flap Seizure}

The main reason for the occurrence of flap seizure failure is that the maximum driving torque provided by the rotary actuator cannot overcome the aerodynamic load and the coupling resistance of the components when the motion law is satisfied. It is not difficult to judge that the required driving torque in the flap drop process is bigger than raise process. According to dynamic analysis, it can be seen that the driving torque reaches the maximum value when the flap deflection angle in the range of $8^{\circ} \sim 12^{\circ}$, and the limit state equation as follows:

\section{$\mathrm{g}(i, \theta)=\lceil T\rceil-|T(i, \theta)|\left(i=1,2,3 \ldots \mathrm{m}, \theta \in\left(8^{\circ}, 12^{\circ}\right)\right)$}

Here $m$ is the Monte Carlo simulation numbers, $\theta$ is the flap deflection angle, $[T]$ is the maximum driving torque that can be provided by the rotary actuator, $T(i, \theta)$ is the maximum driving torque when the flap deflection angle in the range of $8^{\circ} \sim 12^{\circ}$ under the i-th sampling condition. At the same amount of wear, the friction coefficient between track and roller contact surface will change significantly due to the differences among lubrication, abrasive grain and temperature. At $\Delta=0.012$, $\mathrm{m}=100000$, stuck reliability of flap mechanism is calculated under different friction coefficient and the results are shown in table 3.

Table 3. Reliability of flap stuck $([T\rceil=9 \mathrm{~N} / \mathrm{m})$

\begin{tabular}{ccc}
\hline Total wear & Coefficient of friction & Reliability \\
\hline & 0.14 & 1 \\
$\Delta=0.012$ & 0.16 & 0.99456 \\
& 0.18 & 0.81690 \\
& 0.20 & 0.25962 \\
& 0.22 & 0.01658 \\
& 0.24 & 0.00014 \\
\hline
\end{tabular}

According to table 3 , the relationship between the coefficient of friction and the probability of failure as shown in Fig. 10

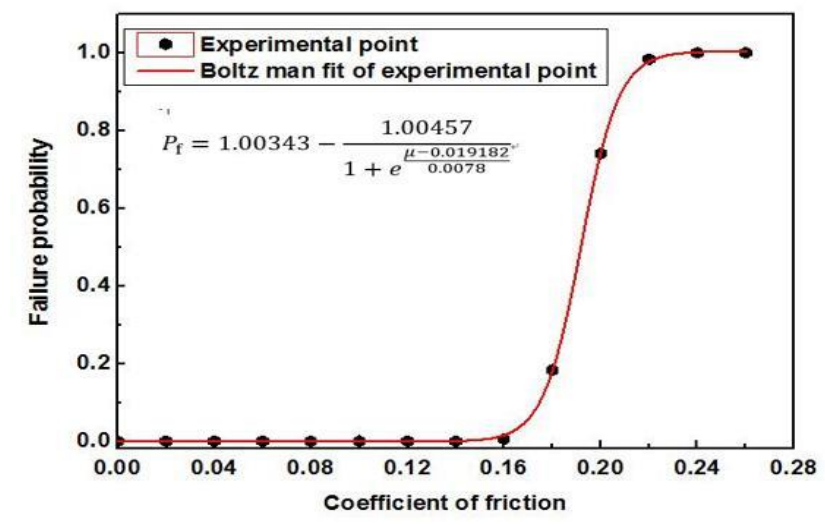

Fig. 10 The curve of failure probability

\section{Summary}

A simulation model of the flap mechanism is established by using Simulink and simultaneous constraint method. Using this model, we can judge the drastically worn part and position of the flap 
mechanism under the influence of comprehensive error. Under dynamic simulation analysis, we find the error of kinematic parameters has obvious regularity and volatility under the influence of synthetic error, they are not as chaotic as we expected. And the flap deflection angle range when the flap defection angle deviation and driving torque reach the maximum are found. Using those results, we can greatly reduce the simulation calculation workload, while we calculate the reliability of flap deflection accuracy and the reliability of flap seizure.

\section{References}

[1]. Huan pang,Tianxiang Yu,Hui Wang,et al. elide rail,ear vector directioning ve torquenge B and $\mathrm{C}$ is wide, Virtual simulation of typical slat mechanism and reliability analysis of kinematic chucking [J] Journal of System Simulation, 2013, 7: 037.

[2]. Bo Yuan,Chenghui feng, gang Li,et al.The dynamic analysis of aircraft flap mechanism [J]. Proceedings of Academic Conference on Structural Strength of China Aeronautical Society, 2013.

[3]. Lijie Cui,Zhenzhou Lu,Feng Zhang et al.Reliability analysis of dynamic response of airplane flap mechanism [J]. Chinese High Technology Letters , 2009, 19 (12): 1299-1304.

[4]. Zhisheng Yi,Jingwu He, Simulation Study on the Design of the flap in civil Aircraft[J]. Aircraft Design, 2010, 30 (1).

[5]. Bo Yuan,Xiaojun Zhu, Numerical analysis of a linkage slide-type trailing edge flap mechanism[J]. Science and Technology Innovation Herald, 2013 (5): 81-82.

[6]. Jiten Patel,Seung-Kyum Choi. Classification approach for reliability-based topology optimization using probabilistic neural networks [J].Structural and Multi-disciplinary Optimization, 2012, v45(4):529-543 